\title{
Biocompatible, Biodegradable Radio-opaque Polymer Nanoparticles.
}

\author{
Vishwas N. Joshi ${ }^{1}$, Henry Smilowitz ${ }^{2}$. \\ 1. Nanoprobes, Incorporated; 95 Horseblock Road, Unit 1; Yaphank, NY 11980. \\ ${ }^{2 .}$ University of Connecticut Health Center, Farmington, CT 06030.
}

The conventional molecular iodine X-ray contrast agents (ICAs) clear rapidly through kidneys and by extravascular diffusion allowing only short imaging times [1]. To address this larger-sized ICAs based on liposomes, polymers, micelles, dendrimers with polyethylene glycol (PEG) core, and metal nanoparticles with longer blood half-lives, or blood-pool agents, that enable targeting and temporal imaging have been developed [2]. Despite varying degrees of success with regards to higher contrast, lower osmolality, longer circulation times and target-specific imaging with these experimental agents the issues related to instability in the physiological medium, toxicity, accumulation in liver and spleen, long-term whole body retention and overall performance remain to be solved [2].

The blood-pool agents that entrap ICAs suffer from "burst release" of contrast agent while others suffer from low iodine loading or low contrast [2]. To extend the blood half-lives of ICAs for target specificand temporal-imaging we used natural and synthetic polymer as delivery vehicles and to reduce kidney burden following burst release that cause adverse reactions in patients with allergies, asthma, kidney diseases and diabetes we covalently linked ICAs to the delivery agents. For good in vivo tolerance and quick transition from pre-clinical to clinical settings we prepared blood-pool ICAs with components that are either FDA (American Food and Drug Administration) approved or generally recognized as safe (or GRAS) by FDA. The functionalized FDA approved ICAs were covalently linked to either GRAS or FDA approved biocompatible/biodegradable naturally occurring carbohydrate polymers, e.g., hyaluronic acid, dextran and cellulose, and synthetic polymers, e.g., aliphatic polyesters, polyanhydrides and polycarbonates [3].

Anionic activation of polylactide, polylactide/glycolide and polycaprolactone followed by reaction with electrophile (grafting method) [4] resulted in variable iodine agent substitution. The iodine loading can be improved by covalently linking ICA derivatives to amine functionalized polylactide, Scheme 1 [5, 6]. However, when the molar ratio of the functionalized (e.g., allyl lactide in Scheme 1)-to-unfunctionalized monomer exceeded 5:95 (5-10\% or more of the functionalized monomer) insoluble blocks were formed. Also, side reaction (e.g., transesterification reaction), products were produced [7]. Therefore, fivemembered planar 1,3-dioxolane-2,4-dione or O-carboxyanhydride (OCA) derivatives were used for polymer preparation, Scheme 2. The OCAs are more reactive and afford polyesters with controlled molecular weights and narrow polydispersities under much milder conditions [7]. Figure 1 shows transmission electron micrograph and micro-computed tomography (micro-CT) contrast produced by polymeric ICA nanoparticles from OCA derivatives (Scheme 2). The new polymeric-ICAs are be evaluated for the specific detection of stenosis in mouse model by micro-CT. 
References:

[1] H. Lusic et al, Chem. Rev., 113 (2013), 1641.

[2] F. Hallouard et al, Biomaterials, 31 (2010), 6249.

[3] B. D. Ulery et al, J. Polym. Sc., Part B: Polym. Phys., 49 (2011), 832.

[4] S. Ponsart et al, Biomacromol., 1 (2000), 275I.

[5] J. Zou et al, Adv. Mater., 23 (2011), 4274.

[6] V. Darcos et al, Polym, Chem, 3 (2012), 362.

[7] O. T. du Boullay et al, J. Am. Chem. Soc., 128 (2006), 16442.

[9] This work was supported by NIH 1 R43 HL117473 SBIR grant.

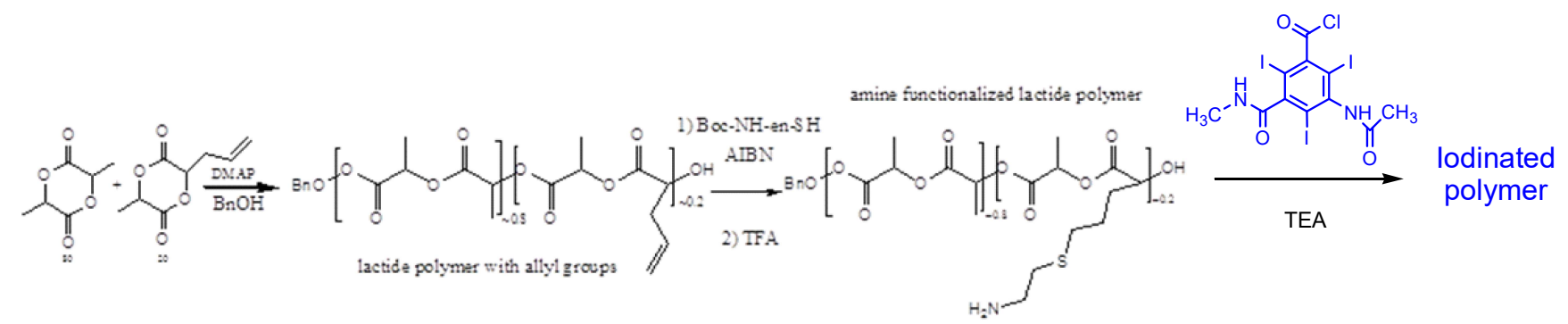

Scheme 1: Amine functionalized polylactide-co-allyl lactide.

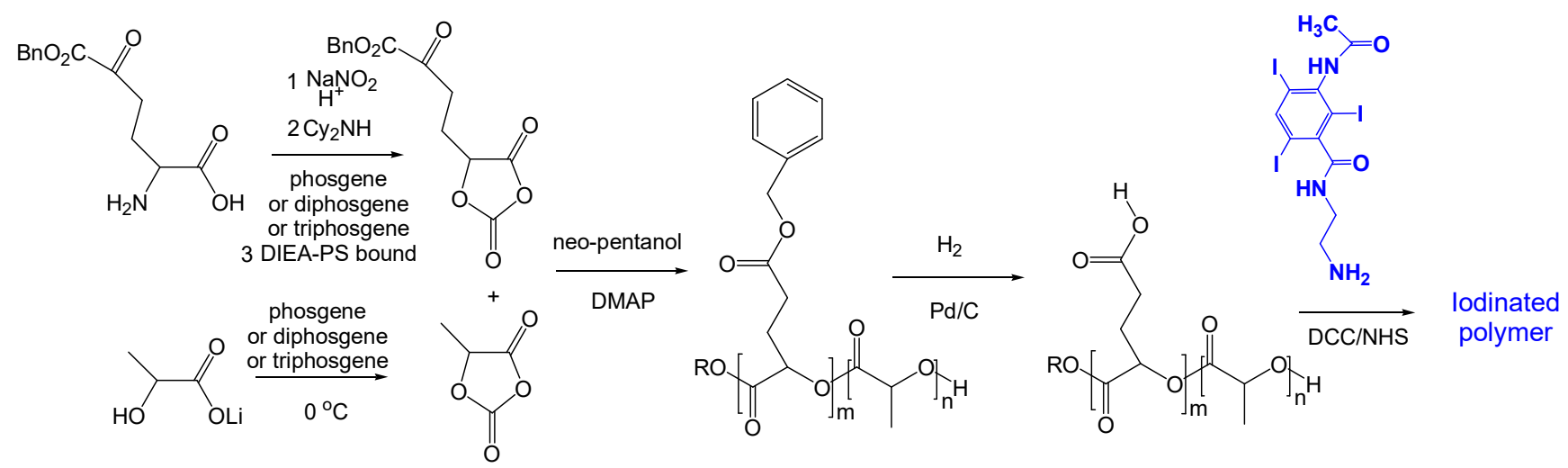

Scheme 2. Preparation of polylactide random co-polymer contrast agent.

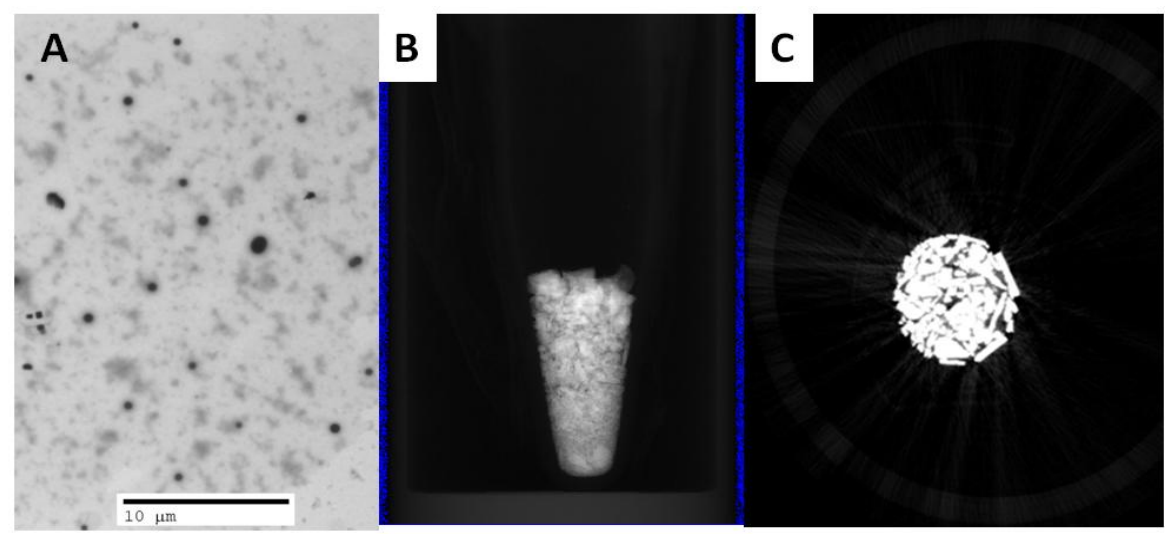

Figure 1. (A) TEM, (B) vertical and (C) horizontal micro-

CT slice through dried iodinated-polymer nanoparticles. 